\title{
Analytical calculation of the frequency-dependent litz wire resistance considering the wire connectors
}

\author{
Daniel Barth, Thomas Leibfried \\ KARLSRUHE INSTITUTE OF TECHNOLOGY \\ Engesserstraße 11 \\ Karlsruhe, Germany \\ Phone: +49 721-60843067 \\ Email: daniel.barth@kit.edu
}

\author{
Giuseppe Cortese \\ DAIMLER AG \\ Hanns-Klemm-Straße 45 \\ Böblingen, Germany
}

\section{Keywords}

$\ll$ Wireless Power Transmission $\gg, \ll$ Contactless Energy Transfer $\gg, \ll$ Charging Infrastructure for EV's $\gg$, $\ll$ Ohmic Losses》

\begin{abstract}
Eddy-current effects have an impact on the resistance of litz wire. They reduce the utilization of the wire cross section. In a certain parameter range, the dominant influencing factor is the current distribution in the wire connectors. The connector itself does not significantly contribute to the wire resistance but it results in an inhomogeneous current distribution among the strands. We quantify the resistance increase by introducing a connector skin-effect factor and give a mathematical derivation. Taking the twisting of the strands into account requires a probabilistic model of the radial strand position. Resistance measurement results validate the calculation approach. We discuss the assumptions and derive the parameter range where the model is valid.
\end{abstract}

\section{Introduction}

Electromobility is a topical issue in electrical engineering and a major field of research. Automobile manufacturers are investing in an increasing number of battery electric vehicle (BEV) models, but the market share of electric vehicles is still low [1]. Aiming at the improvement of consumer acceptance of electric vehicles, we consider the charging process. A BEV must be charged frequently compared to refueling an automobile with a combustion engine. Wireless power transfer (WPT) is a promising technology for overcoming this constraint.

- Stationary inductive WPT makes charging more convenient during periods of rain or snowfall, as handling of the cable is not necessary. The technology can also prevent the user from forgetting to plug in the connector at home. It is safe and long-lasting, as there are no open contacts and no movable parts. Additionally, WPT is necessary for self-driving cars.

- Dynamic inductive WPT enables electric vehicles to be charged while in motion. This technology could overcome the problem of limited range if it is applied in the road surfaces of motorways. While BEVs are well suited to driving in urban areas, they are not competitive for long-distance journeys. Dynamic charging reduces the required battery capacitance and can save cost, weight and space in a vehicle. Compared to conductive dynamic charging technologies, dynamic WPT is robust, due to the lack of movable parts. It can be applied to all kinds of vehicles.

Inductive WPT systems, both stationary and dynamic, consist of magnetically coupled coils. The typical operational frequency is between $81.38 \mathrm{kHz}$ and $90.00 \mathrm{kHz}$ [2], and hence the coils are made of litz wire to reduce eddy-current losses. Litz wire is a major cost factor due to the complexity of its manufacturing process, and it has a significant influence on the WPT systems efficiency. As high efficiency and low cost are important objectives, calculation of the frequency-dependent resistance of the coils is necessary. 
Finite element analysis (FEA) is commonly used in the coil design for WPT systems. Modeling litz wire using FEA software is possible in the case of short sections of wire, where the wire consists of a manageable number of strands, (e.g., [3]). If the whole coil has to be modeled, the combination of a high number of very thin and very long strands exceeds common computing capacities [4]. In this work, FEA serves as a verification tool but we focus on analytical resistance calculations.

The calculation of eddy-current effects, as will be described in the next chapter, is necessary in order to add a frequency-dependent part to the DC resistance. In the literature, several publications describe the analytical calculation of skin effects and proximity effects in cylindrical strands, (e.g., [5], [6]). The influences of strand twisting, bundle formation, non-ideal insulation of litz wire and material choice, are described. Further investigations show that the wire connectors must be considered [7]. This theory replaces the assumption that each strand in the litz wire carries the same current with the assumption that a strand carries a certain current according to its position in the connector. Hence the authors can explain deviations in measurement and calculation results that arise, especially in the case of litz wire, for high-current applications. The quantification of the frequency-dependent change of resistance due to this effect is achieved using a set of 3D and 2D FEA simulations. This method is time-consuming and requires FEA software, while eddy-current effects in the strands can be calculated in a numerical computing environment [5]. Therefore, we introduce a new method for the calculation of the inhomogeneous current distribution in litz wire, caused by the connectors. This enables a more accurate prediction of the frequency-dependent resistance, without using FEA software.

\section{Eddy-Current Effects}

Eddy-current losses in a conductor arise due to an inhomogeneous current density. In other words, eddycurrent effects lead to a non-ideal utilization of the conductor cross section, which can be expressed as a rise in the conductor resistance. We distinguish two different effects:

- The skin effect, which describes the current distribution as a result of the interaction with the magnetic field generated by the current itself.

- The proximity effect, which describes the influence of external magnetic fields on the current distribution in a conductor.

The influence of the connectors on the litz wire resistance depends on the magnetic field affecting the connectors. Both the skin effect and the proximity effect must be considered. However, if we place the connectors at a distance from the coil windings, the external magnetic field is negligible. The skin effect dominates in the current distribution. Therefore, we can omit the connector proximity effect.

\section{Skin Effect}

To investigate the skin effect we must calculate the current density in a cylindrical conductor. From Maxwell's equations, we obtain the diffusion equation of the electric field $\vec{E}$,

$$
\Delta \vec{E}=\mu \sigma \frac{d \vec{E}}{d t}
$$

where $\mu$ is the permeability, $\sigma$ is the conductivity and $\Delta$ is the Laplace operator. Inserting $\sigma \vec{E}=\vec{J}$ and the direction of the current in cylindrical coordinates $\vec{J}=J_{z} \cdot \vec{e}_{z}$, and assuming the current to be sinusoidal according to $I_{z}(t)=\hat{I} \cdot e^{j \omega t}$, we rewrite the differential equation as:

$$
\frac{\partial^{2}}{\partial r^{2}} J_{z}+\frac{1}{r} \cdot \frac{\partial}{\partial r} J_{z}-j \omega \mu \sigma \cdot J_{z}=0
$$

The constants can be combined in a new constant $\zeta=\sqrt{-j \omega \mu \sigma}$, which we insert in (2):

$$
\frac{\partial^{2}}{\partial r^{2}} J_{z}+\frac{1}{r} \cdot \frac{\partial}{\partial r} J_{z}+\zeta^{2} \cdot J_{z}=0
$$


As this is Bessel's differential equation, it can be solved using the following function.

$$
J_{z}(r)=\frac{I_{0} \cdot \zeta}{2 \pi R_{0}} \cdot \frac{g_{0}(\zeta \cdot r)}{g_{1}\left(\zeta \cdot R_{0}\right)}
$$

where $I_{0}$ is the current through the conductor, $g_{0}$ and $I_{1}$ are Bessel functions of the first kind and $R_{0}$ is the conductor radius. $J_{z}(r)$ denotes the current density in the conductor depending on the radial position $r$. The frequency-dependent $\mathrm{AC}$ resistance is:

$$
R_{a c}=\Re\left(\frac{U_{a c}}{I_{a c}}\right)=\Re\left(\frac{\int_{0}^{l} \sigma^{-1} J_{z}\left(R_{0}\right) d z}{\int_{0}^{2 \pi} \int_{0}^{R} J_{z}(r) \cdot r d r d \phi}\right)=\Re\left(\frac{l \cdot \zeta \cdot g_{0}\left(\zeta \cdot R_{0}\right)}{\sigma \cdot 2 \pi R_{0} g_{1}\left(\zeta \cdot R_{0}\right)}\right)
$$

where $l$ is the length of the conductor. We divide (5) by the DC resistance

$$
R_{d c}=\frac{l}{\sigma \pi R_{0}^{2}}
$$

to obtain the skin-effect factor $F$.

$$
F=\frac{R_{a c}}{R_{d c}}=\Re\left(\frac{R_{0} \cdot \zeta}{2} \cdot \frac{g_{0}\left(\zeta \cdot R_{0}\right)}{g_{1}\left(\zeta \cdot R_{0}\right)}\right)
$$

In addition to the notation in (7), we need another notation for the skin-effect factor, based on the power dissipation in the conductor. This shows that the skin-effect factor represents the variance of the current density distribution. Using the power dissipation density $p=\vec{E} \cdot \vec{J}^{*}=\sigma^{-1} \cdot J_{z} J_{z}^{*}=\sigma^{-1} \cdot\left|J_{z}\right|^{2}$, we derive the following.

$$
F=\frac{P_{a c}}{P_{d c}}=\frac{\frac{1}{\sigma} \int_{v}\left|J_{z}(r)\right|^{2} d v}{\frac{l}{\sigma \pi R_{0}^{2}} \cdot I_{0}^{2}}=\frac{\frac{2 \pi \cdot l}{\sigma} \int_{0}^{R_{0}}\left|J_{z}(r)\right|^{2} \cdot r d r}{\frac{2 \pi \cdot l}{\sigma} \cdot\left(\frac{I_{0}}{\pi \cdot R_{0}^{2}}\right)^{2} \cdot \frac{R_{0}^{2}}{2}}=\frac{\int_{0}^{R_{0}}\left|J_{z}(r)\right|^{2} \cdot r d r}{\left(\frac{I_{0}}{\pi \cdot R_{0}^{2}}\right)^{2} \cdot \frac{R_{0}^{2}}{2}}
$$

where $v$ is the conductor volume. Here, $F$ represents the square of the AC current density divided by the square of the DC current density. (7) and (8) give exactly equivalent results.

\section{Proximity Effect}

Although we focus on the skin effect in the connectors, the proximity effect in the strands must also be considered, to complete the analytical model and to compare it with measurement data. If a homogeneous magnetic field $H(t)=\hat{H} \cdot e^{j \omega t}$ affects a cylindrical conductor with length $l$, it induces an electric field which causes eddy currents. Integration of the eddy-current losses in the conductor volume gives the proximity-effect loss in a single strand:

$$
P_{p}=2 \cdot G \cdot\left(H_{i n t, r m s}^{2}+H_{\text {ext }, r m s}^{2}\right) \cdot l
$$

where $H_{\text {int }}$ is the magnetic field caused by other strands in the same winding and $H_{\text {ext }}$ is the magnetic field caused by other windings and coils [4], [6]. The factor $G$ depends on the relations between conductor diameter, conductivity and permeability and the frequency of the field. It can be derived analytically [5], [8]. The additional resistance caused by the proximity effect is $R_{p}=\frac{P_{p}}{I_{0}^{2}}$.

\section{Frequency-Dependent Resistance of Litz Wire}

Common litz wire models use geometrical and material data to calculate the skin effect and the proximity effect in the strands. It is possible to perform the simulations in a numerical computing environment [9]. For comparing simulation and measurement data, we use a coil with 10 windings, made of high-power litz wire with 7,350 insulated strands. Each strand has a diameter of $71 \mu \mathrm{m}$. Fig. 1 shows the measurement setup. The OMICRON Bode 100 serves as the measurement device. It operates in external bridge 
a)

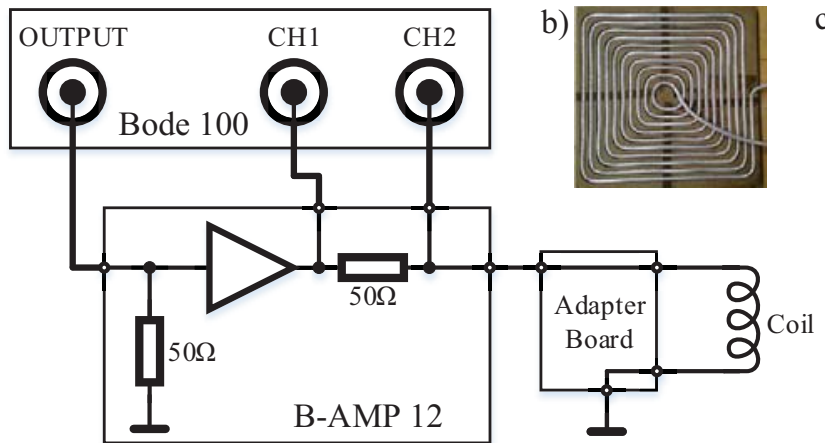

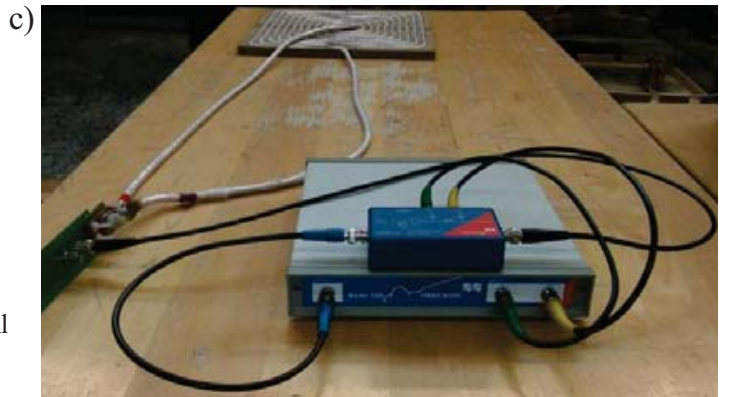

Fig. 1: a) Circuit diagram of the measurement setup. b) The test coil consists of 10 windings in the shape of a square. The edge length is $380 \mathrm{~mm}$. c) Picture of the measurement setup. The power supply cables have been removed.

mode, where the OMICRON B-AMP 12 is the external bridge. The coil is connected to the B-AMP via an adapter board that converts BNC to cable lug. Note that the connectors are at a distance of more than twice the coil edge length from the outermost winding, to avoid proximity-effect losses. As the coil resistance is very small, the accuracy of the measurement setup is not good enough to determine the absolute resistance. Therefore, only the frequency-dependent gradient will be evaluated. Fig. 2 displays the resistance measurements after warm-up and calibration of the Bode 100. We compare this to a simulation that takes eddy-current effects in the strands into account. The thick vertical lines divide the

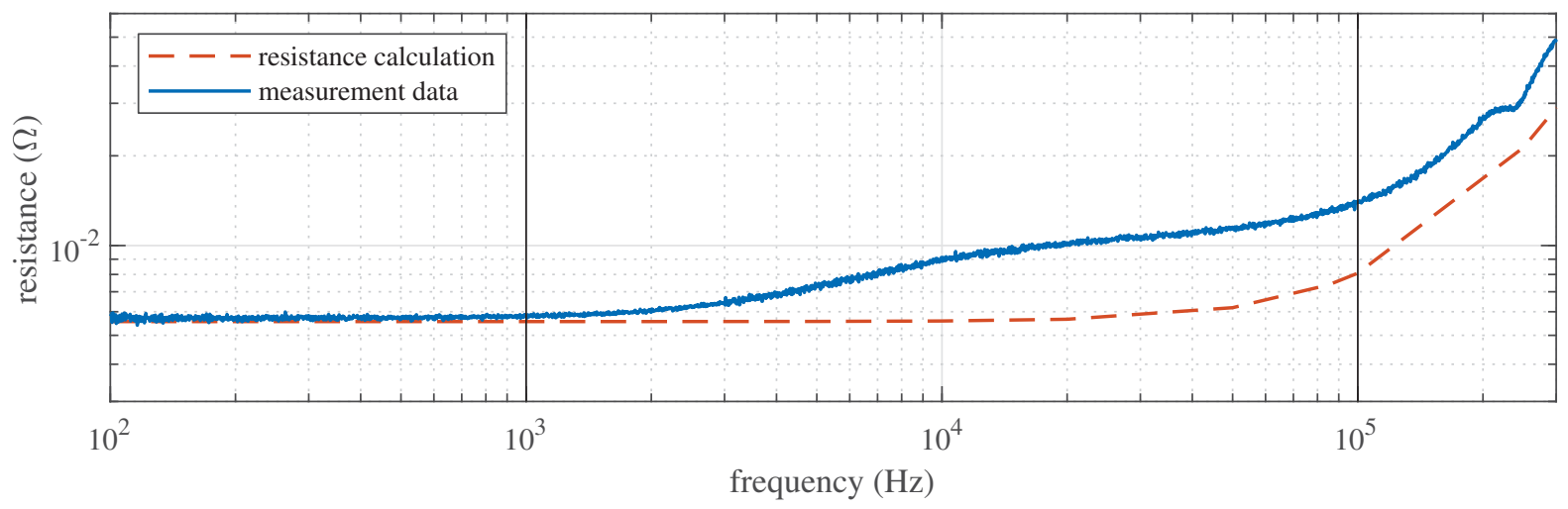

Fig. 2: Resistance of a WPT coil as a function of frequency. The calculation does not consider the connectors.

diagram into three sections:

1. In the first section, up to $1 \mathrm{kHz}$, only the $\mathrm{DC}$ resistance is visible.

2. In the second section, the measured resistance increases with the frequency. The model does not cover this effect.

3. The third section is dominated by the proximity effect. Both curves have a similar progression. Due to the small strand diameter in this design, the skin effect in the strands does not occur. According to [7], the difference seen in the second section results from the skin effect in the wire connectors. The current in each strand is defined by its position in the connectors. There is no redistribution in the coil, due to the insulation between the strands. The increase in the resistance due to the skin effect in the connector itself can be neglected, as the connector is short. However, its effect on the current distribution in the strands is measurable, as the strands are much longer and therefore have a much higher resistance. The litz wire measured in Fig. 2 was made for high-current applications. Its conductor cross section of $29.1 \mathrm{~mm}^{2}$ implies that the conductor diameter is larger than the skin depth in the frequency range above $1 \mathrm{kHz}$. The connectors must have at least the same cross section. Thus the skin effect in the connectors is a likely explanation for the deviation in the second section of Fig. 2. 


\section{Modeling the Influence of the Wire Connectors}

To perform the design of high-current litz wire in a numerical computing environment, we propose a new method for calculating the resistance increase due to the connectors. It is based on the observations in the previous section and the following assumptions:

- The current density in each strand is the mean value of the current densities at the positions of its contact with the connectors.

- The connectors have the shape and current density distribution of a cylindrical conductor.

A factor which represents the resistance increase due to the connector skin effect can be calculated if the current densities at the ends of each strand are known. However, for a single strand, it is impossible to calculate its radial positions at the connectors precisely. The strands are twisted into the shape of a non-ideal helix in multiple layers, and the wire is itself curved and twisted. Given the high number of strands, we assume the radial position of a strand to be a random variable with a certain distribution function. Probability theory cannot provide information about a single event, but it is possible to reliably predict the deviance and the expected value for a large number of events. Therefore, we treat the radial position of a strand as an event that is connected with a particular current density. The variance of a random variable describing the current density distribution of the strands yields information on how the current densities in the strands differ from the expected value. The skin-effect factor in (8) contains the same information. Calculation of the skin-effect factor of a single cylindrical conductor using probability theory will verify this approach.

\section{The Skin-Effect Factor in Probability Theory}

\section{Cylindrical Conductors}

Considering the radial position inside a homogeneous cylindrical conductor as a random variable $R$, we derive its cumulative distribution function:

$$
F_{R}(r)=P(R \leq r)= \begin{cases}0 & r<0 \\ \frac{\pi r^{2}}{\pi R_{0}^{2}} & 0 \leq r \leq R_{0} \\ 1 & r>R_{0}\end{cases}
$$

and its probability density function:

$$
f_{R}(r)=\frac{d}{d r} F_{R}(r)= \begin{cases}\frac{2 r}{R_{0}^{2}} & 0 \leq r \leq R_{0} \\ 0 & \text { else }\end{cases}
$$

By inserting $R$ in (4) we obtain the random variable $J=J_{z}(R)$, which describes the current density probability depending on the radial position. The law of the unconscious statistician enables the calculation of its expected value.

$$
\mathrm{E}[J]=\mathrm{E}\left[J_{z}(R)\right]=\int_{-\infty}^{\infty} J_{z}(r) \cdot f_{R}(r) d r=\frac{I_{0}}{\pi R_{0}^{2}}
$$

The expected value must be equal to the DC current density $J_{0}=\frac{I_{0}}{\pi R_{0}^{2}}$. For demonstration purposes, Fig. 3 shows the normalized probability density function and the normalized current density. Increasing the frequency increases the current density near $R_{0}$, which increases the variance of $J=J_{z}(R)$. We derive the variance as:

$$
\operatorname{Var}(J)=\mathrm{E}\left[|J-\mathrm{E}[J]|^{2}\right]=\int_{-\infty}^{\infty}\left|J_{z}(r)-\mathrm{E}[J]\right|^{2} \cdot f_{R}(r) d r
$$




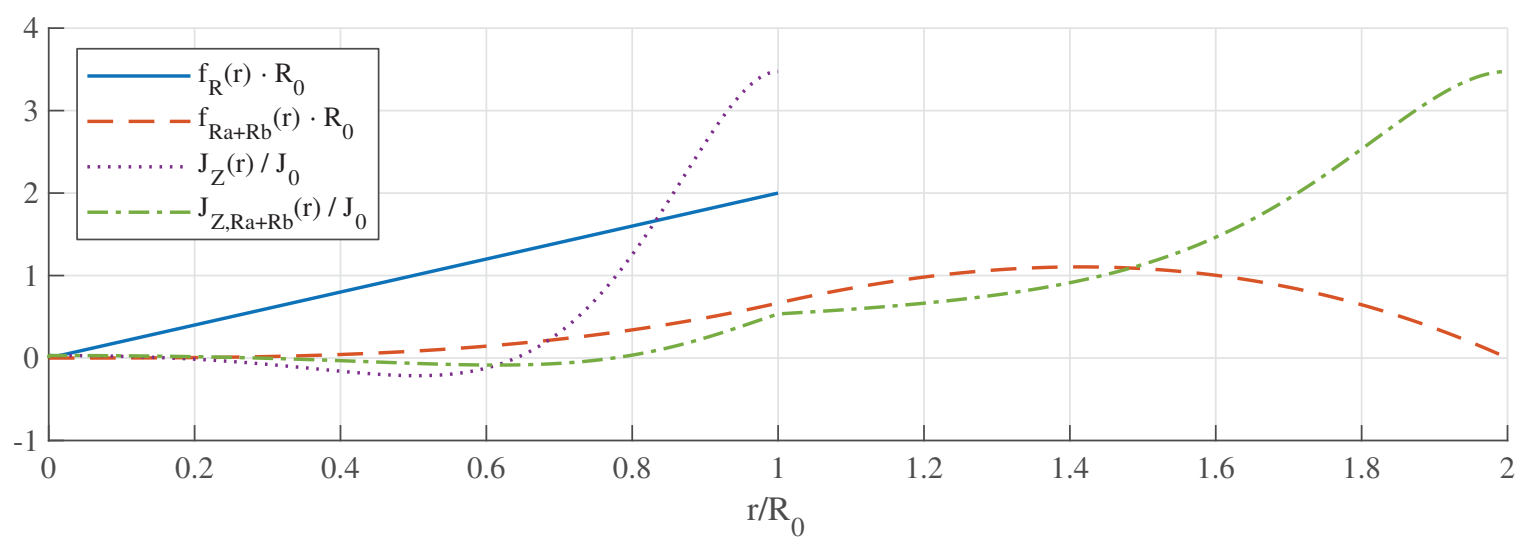

Fig. 3: Normalized probability density and current density functions of a solid cylindrical conductor and of litz wire with cylindrical connectors.

which represents the squared deviation of $J$ from the expected value $\mathrm{E}[J]$. The similarity to (8) leads to another notation for the skin-effect factor:

$$
F=\frac{\operatorname{Var}(J)+\mathrm{E}[J]^{2}}{\mathrm{E}[J]^{2}}=\int_{-\infty}^{\infty}\left|\frac{J_{z}(r)}{\mathrm{E}[J]}-1\right|^{2} \cdot f_{R}(r) d r+1
$$

which always gives the same result as (7) and (8).

\section{Litz Wire with Cylindrical Connectors}

This calculation method can be applied to litz wire with a sufficiently high number of strands, with a connector on each side. As both connectors have an influence on the current density in each strand, the current density is an event that depends on the combination of two random variables $R_{a}$ and $R_{b}$. A mutual probability density function is required. Due to the non-ideal twisting, we consider the radial positions of a strand at each connector to be independent. The probability density function of the sum of two independent random variables $R_{a}$ and $R_{b}$ is the convolution of their density functions, therefore

$$
f_{R_{a}+R_{b}}(r)=\left(f_{R_{a}} * f_{R_{b}}\right)(r)= \begin{cases}\int_{0}^{r} \frac{2 \tau}{R_{0}^{2}} \cdot \frac{2(r-\tau)}{R_{0}^{2}} d \tau & 0<r \leq R_{0} \\ \int_{r-R_{0}}^{R_{0}} \frac{2 \tau}{R_{0}^{2}} \cdot \frac{2(r-\tau)}{R_{0}^{2}} d \tau & R_{0}<r \leq 2 R_{0} \\ 0 & \text { else }\end{cases}
$$

is the probability density function describing the independent choice of two radial positions at the connectors. As for $R_{a}+R_{b}$, a random variable representing the current density depending on both connector positions is required. To calculate its expected value and variance, we define the function $g$, which determines the random variable $J_{R_{a}+R_{b}}$ depending on $R_{a}+R_{b}$ :

$$
J_{R_{a}+R_{b}}=g\left(R_{a}+R_{b}\right)
$$

We assume that the current density in each strand is equal to the average value of the current densities at its ends in the connectors. Since it depends on the sum of two radial positions $R_{a}+R_{b}$, the function $g$ must contain all possible combinations of current densities corresponding to that value. Therefore the average values of each combination are added, and the result is divided by the number of summands. Transferring this procedure to an integral notation, we define

$$
g(r)=J_{z, R_{a}+R_{b}}(r)= \begin{cases}\frac{1}{2 r} \int_{0}^{r} J_{z}(\tau)+J_{z}(r-\tau) d \tau & 0<r \leq R_{0} \\ \frac{1}{2\left(2 R_{0}-r\right)} \int_{r-R_{0}}^{R_{0}} J_{z}(\tau)+J_{z}(r-\tau) d \tau & R_{0}<r \leq 2 R_{0} \\ 0 & \text { else }\end{cases}
$$


as the current density depending on the radial position $r=r_{a}+r_{b}$. Inserting the random variable $J_{R_{a}}+R_{b}$ in (12) - (14), we derive the connector skin-effect factor

$$
F_{c o n}=\frac{\operatorname{Var}\left(J_{R_{a}+R_{b}}\right)+\mathrm{E}\left[J_{R_{a}+R_{b}}\right]^{2}}{\mathrm{E}\left[J_{R_{a}+R_{b}}\right]^{2}}=\int_{-\infty}^{\infty}\left|\frac{J_{z, R_{a}+R_{b}}(r)}{\mathrm{E}\left[J_{R_{a}+R_{b}}\right]}-1\right|^{2} \cdot f_{R_{a}+R_{b}}(r) d r+1
$$

which represents the resistance increase due to the unequal current distribution among the strands.

Fig. 3 shows the normalized functions $f_{R_{a}+R_{b}}(r) \cdot R_{0}$ and $J_{z, R_{a}+R_{b}}(r) / J_{0}$. The maximum current density is equal to that of a solid conductor with the same diameter. However, due to the redistribution of the strands, the radial positions with high current density have a lower probability. Compared with a solid conductor, the skin effect of the litz wire is much lower.

\section{Comparison of Simulation and Measurement Data}

A comparison of the measurement data already introduced in Fig. 2 and the resistance calculation considering the connectors, validates the new method. Fig. 4 shows that the frequency-dependent resistance curve is the sum of the DC resistance and the additional resistance caused by the skin effect and the proximity effect. Between $1 \mathrm{kHz}$ and $100 \mathrm{kHz}$, where Fig. 2 shows a disagreement between measurement and simulation, the new method leads to a better match. We can cross-check the connector-effect theory by

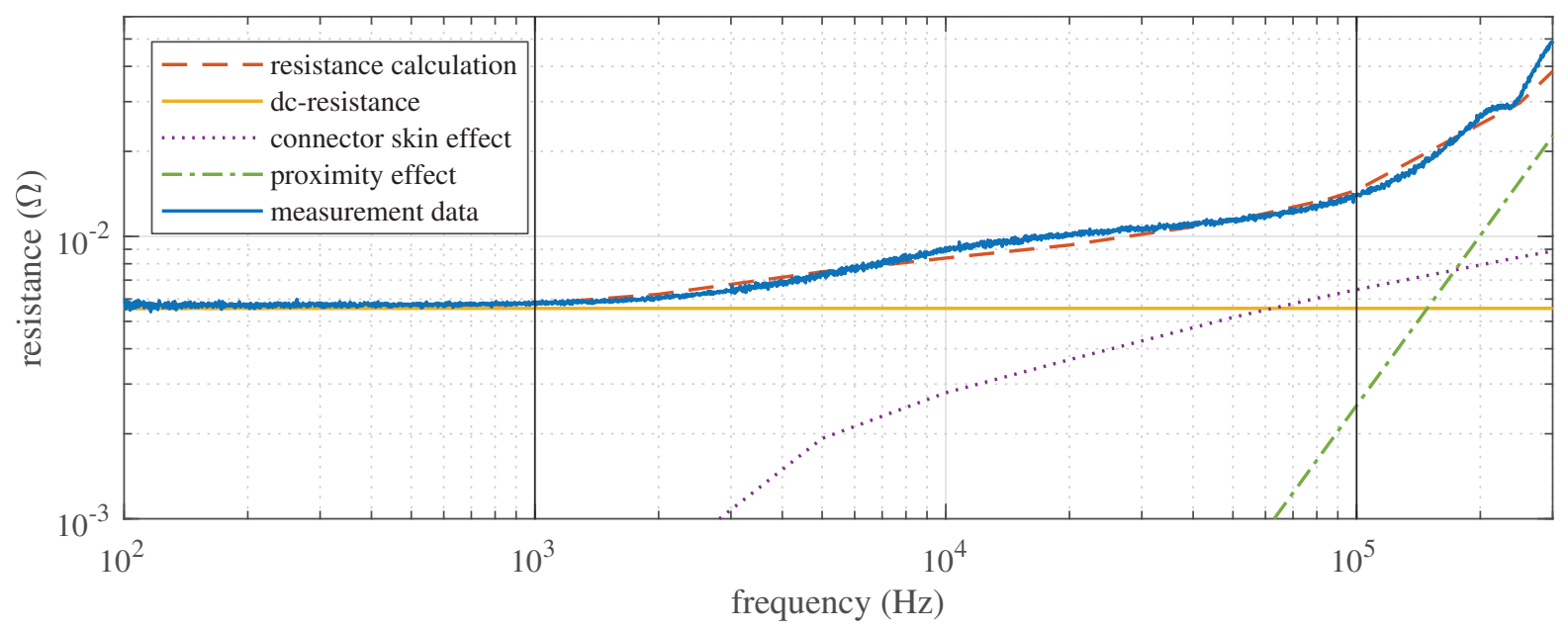

Fig. 4: The new resistance calculation conforms to the measurement curve. The overall resistance is the sum of the DC resistance and the additional resistance caused by the skin effect and the proximity effect.

resistance measurements and calculations for a coil that consists of litz wire with 512 insulated strands, where each strand has a diameter of $100 \mu \mathrm{m}$. Compared with the wire in Fig. 4, the copper cross section is much smaller, and therefore we expect the connector skin effect to be less significant. Fig. 5 shows that both measurement and simulation fulfill the expectation.

\section{Model Discussion}

\section{Application Range}

To examine the parameter range where consideration of the connectors is necessary, we calculate $F_{c o n}$ as a function of $R_{0} / \delta$, where $\delta=(1-j) / \zeta$ is the skin depth. Fig. 6 shows the gradient of $F_{c o n}$ and the skin-effect factor of a solid conductor $F$. $F_{\text {con }}$ clearly has a significant influence if the skin depth is below $R_{0} / 2$. Compared to $F$, it is less steep, which means that the litz wire has a much lower AC resistance than a solid conductor with the same cross section. In Fig. 4, the region where the connector effect is dominant in the resistance measurement $(1 \mathrm{kHz} \ldots 100 \mathrm{kHz})$ is equivalent to $R_{0} / \delta=1.4 \ldots 14$. 


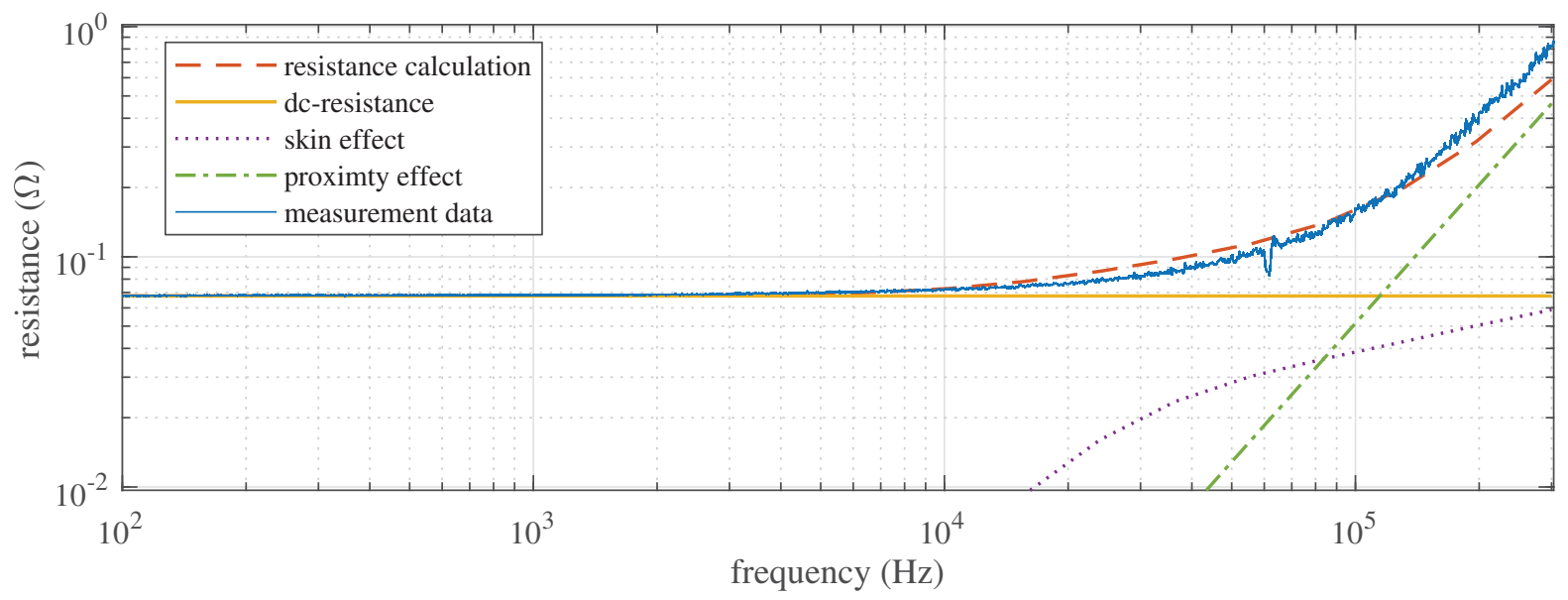

Fig. 5: Compared with Fig. 4, the connector skin effect is negligible in this litz wire design. Its copper cross section is much smaller.

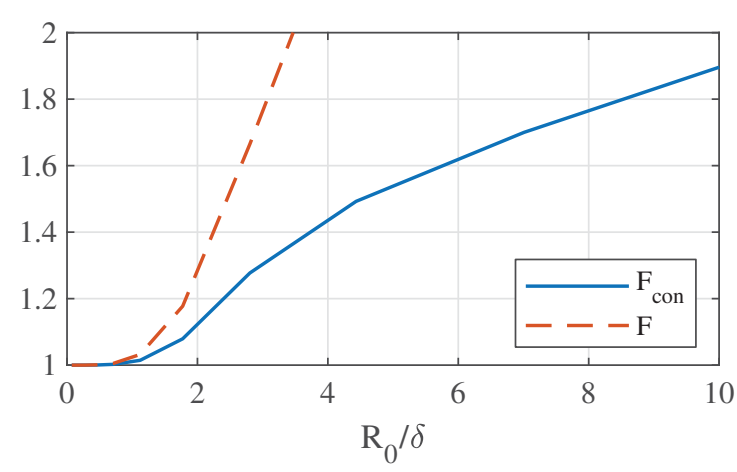

Fig. 6: Gradient of the connector skin-effect factor $F_{c o n}$ and the skin-effect factor $F$, depending on the conductor radius and the skin depth.

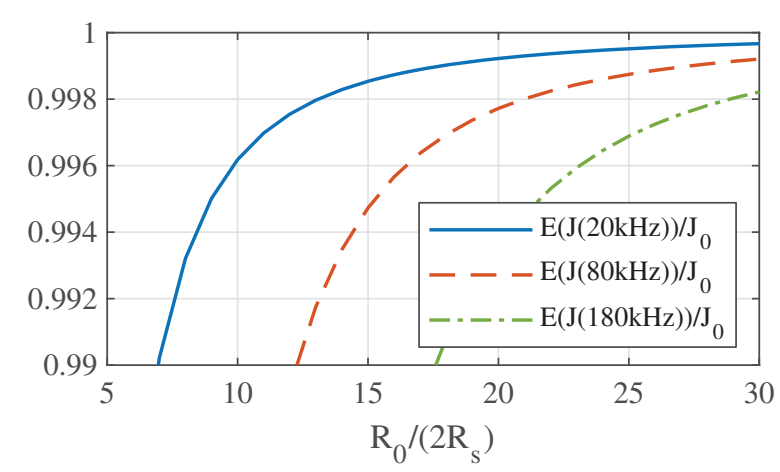

Fig. 7: Deviation of the current density expected value from the homogeneous current density, in the case of discrete strand positions.

\section{Limitations}

\section{Relation Between Strand Diameter and Wire Diameter}

It is necessary to understand the requirements and the limitations of the model. The density functions are a homogeneous representation of discrete strand positions. The homogenization is valid only if the strand diameter is small enough compared to the wire diameter. We investigate the difference between the homogeneous and the discrete current densities by approximating (12) with the Riemann integral:

$$
\mathrm{E}[J]=\mathrm{E}\left[J_{z}(R)\right]=\sum_{k=0}^{n-1} J_{z}\left(r_{k}\right) \cdot f_{R}\left(r_{k}\right) \cdot \frac{R_{0}}{n}
$$

where $n=R_{0} /\left(2 R_{S}\right) . R_{s}$ represents the radius of a strand. Like $\mathrm{E}[J]=J_{0}$ in the homogeneous case, the Riemann integral calculation of $\mathrm{E}[J] / J_{0}$ gives the dependence of $J_{z}(r)$ on $n$. Fig. 7 shows that the deviation is frequency-dependent. The high current density at the outer edge of the connector appears in the litz wire only if the strands are small enough. However, to reduce the strand-level skin effect this condition will probably be fulfilled. The litz wire used in the measurement setup (Fig. 1) has a ratio of $R_{0} /\left(2 R_{S}\right)>42$. 


\section{Connector Shape}

The derivation of the connector skin-effect factor in (18) is valid for rotationally symmetric connectors only. It is possible to generalize the calculation and consider any kind of connector shape.

$$
F_{\text {con }}=\frac{\operatorname{Var}\left(J_{X_{a}+X_{b}}\right)+\mathrm{E}\left[J_{X_{a}+X_{b}}\right]^{2}}{\mathrm{E}\left[J_{X_{a}+X_{b}}\right]^{2}}=\int_{-\infty}^{\infty}\left|\frac{J_{z, X_{a}+X_{b}}\left(x_{1}, \ldots, x_{n}\right)}{\mathrm{E}\left[J_{X_{a}+X_{b}}\right]}-1\right|^{2} \cdot f_{X_{a}+X_{b}}\left(x_{1}, \ldots, x_{n}\right) d x_{1} \ldots d x_{n}+1
$$

where $X_{1}, \ldots, X_{n}$ are random variables with the combined probability density function $f_{X}\left(x_{1}, \ldots, x_{n}\right)$. Instead of depending on the radius only, the current density will depend on multiple dimensions.

Alternatively, it is possible to consider the shape by introducing a correction factor. Fig. 8 shows a common cable lug. This connects the strands in a rectangular or stadium shape. If the skin depth is
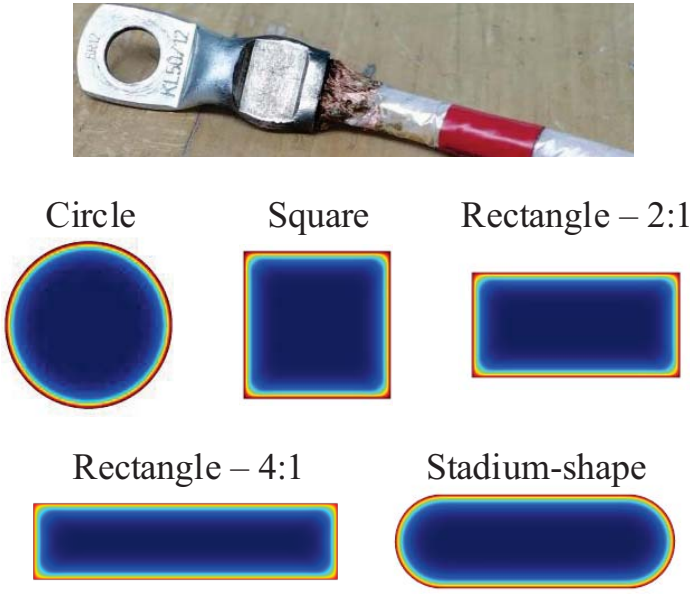

Fig. 8: The current distribution inside a cable lug (picture) can be approximated by various geometrical shapes.

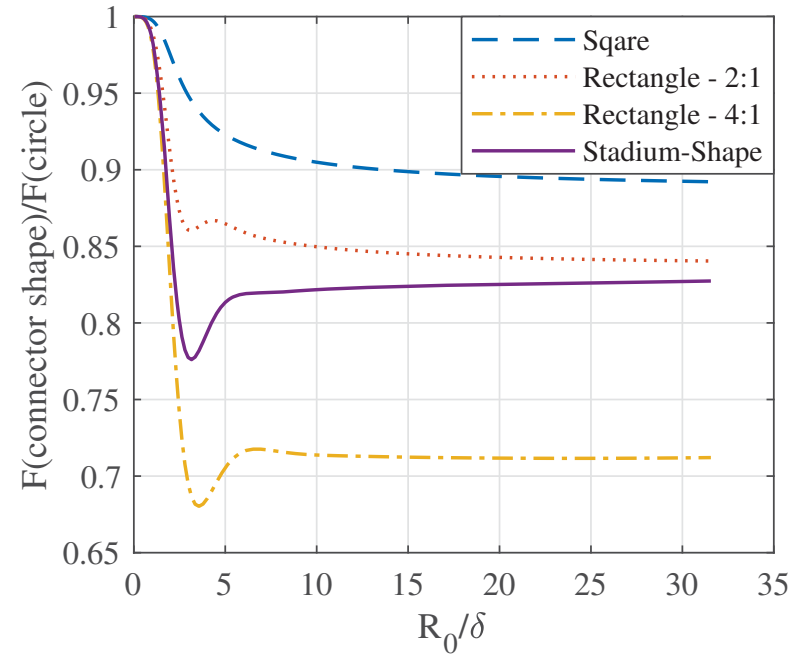

Fig. 9: Dividing the skin-effect factor of the shapes in Fig. 8 by the skin-effect factor of a circular conductor gives a stationary final value.

much smaller than the edge length, most of the current flow is located on the conductor surface. Fig. 8 displays basic shapes with identical cross sections. We compare the scaled shape perimeter $\gamma=2 \pi r / p$ in Table I with the scaled skin-effect factor $F_{\text {shape }} / F_{\text {circle }}$ in Fig. 9. If $R_{0}>>\delta$ is true, the factor $\gamma$ acts as

Table I: Comparison of conductor shape perimeters

\begin{tabular}{cccccc}
\hline & Circle & Square & Rectangle - 2:1 & Rectangle - 4:1 & Stadium shape - 2:1 \\
\hline$p$ & $2 \pi r$ & $4 r \sqrt{\pi}$ & $3 r \sqrt{2 \pi}$ & $5 r \sqrt{\pi}$ & $(8+2 \pi) r \sqrt{\frac{\pi}{8+\pi}}$ \\
$\frac{2 \pi r}{p}$ & 1 & 0.886 & 0.836 & 0.709 & 0.828 \\
\hline
\end{tabular}

a correction factor. In the derivation of $F$ and $F_{c o n}$, we can apply this by defining $J_{z, \gamma}(r)=\gamma \cdot J_{z}(r)$ and $f_{R, \gamma}(r)=\frac{1}{\gamma} \cdot f_{R}(r)$.

\section{Interaction of Different Eddy-Current Effects}

The resistance calculation in Fig. 4 sums the resistance fractions caused by the various effects, without considering any interactions. This is a common practice, as the orthogonality of the skin effect and the proximity effect in the strands is proved in [5]. However, the strand-level proximity-effect model is based on the assumption that the winding is a region of uniform current density [6]. As the currents through the strands actually differ due to the connectors, this assumption is valid only if the twisting continuously reallocates the adjacent strands. In this case, the current density in a small bundle of strands is uniform 
throughout the wire, and the connector skin effect does not influence the average proximity effect in the strands.

In a WPT application, the connectors will probably be much closer to the coil than in Fig. 1. If there is no proper electromagnetic shielding, the proximity effect will influence the current distribution in the connectors. Consideration of the connector proximity effect will further improve the litz wire model.

\section{Conclusion}

The frequency-dependent current distribution among the insulated strands of litz wire is unequal, due to eddy-current effects in the wire connectors. We focus on the AC resistance calculation including consideration of the connector skin effect. High-power litz wire, consisting of thousands of strands, appears to have a significant resistance increase in the frequency range where the skin depth is $1.4 \ldots 14$ times smaller than the connector radius. The high number of non-ideal twisted strands enables a new calculation method. This uses probability theory to describe the radial position of the strands. Applying the probabilistic strand positions in the current density calculation provides the connector skin-effect factor, which depends on the current density variance. This gives the resistance increase of the litz wire, depending on the DC resistance.

Following the mathematical derivation, the model discussion shows that the assumption of a homogeneous probability density function for the strand position is valid in the parameter range where the connectors have a significant influence on the wire resistance. Resistance measurement results validate the new calculation method in the case of litz wire made for high-power WPT applications. Further improvement can be achieved by considering the connector proximity effect.

\section{References}

[1] Bunsen T. et al.: Global EV Outlook 2018, IEA Publications, International Energy Agency, May 2017

[2] SAE International: Technical Information Report J2954, Wireless Power Transfer for Light-Duty Plug-In/ Electric Vehicles and Alignment Methodology, May 2016

[3] Plumed E., Acero J., Lope I. and Carretero C.: 3D Finite Element Simulation of Litz Wires with Multilevel Bundle Structure, IECON 2018 - 44th Annual Conference of the IEEE Industrial Electronics Society, Washington, DC, 2018, pp. 3479-3484

[4] Nan X. and Sullivan C.R.: An improved calculation of proximity-effect loss in high-frequency windings of round conductors, IEEE 34th Annual Conference on Power Electronics Specialist, 2003. PESC '03., Acapulco, Mexico, 2003, pp. 853-860 vol.2

[5] Ferreira J. A.: Analytical computation of AC resistance of round and rectangular litz wire windings, in IEE Proceedings B - Electric Power Applications, vol. 139, no. 1, pp. 21-25, Jan. 1992

[6] Sullivan C. R. and Losses L.: Analytical model for effects of twisting on litz-wire losses, 2014 IEEE 15th Workshop on Control and Modeling for Power Electronics (COMPEL), Santander, 2014, pp. 1-10

[7] Roßkopf A., Bär E. and Joffe C.: Influence of Inner Skin- and Proximity Effects on Conduction in Litz Wires, in IEEE Transactions on Power Electronics, vol. 29, no. 10, pp. 5454-5461, Oct. 2014

[8] Sullivan C. R.: Computationally efficient winding loss calculation with multiple windings, arbitrary waveforms, and two-dimensional or three-dimensional field geometry, in IEEE Transactions on Power Electronics, vol. 16, no. 1, pp. 142-150, Jan. 2001

[9] Barth D., Klaus B. and Leibfried T.: Litz wire design for wireless power transfer in electric vehicles, 2017 IEEE Wireless Power Transfer Conference (WPTC), Taipei, 2017, pp. 1-4 